Acta Poetica 27 (1)

PRIMAVERA

2006

\title{
Yo no es un O/otro
}

\author{
M.-Pierrette Malcuzynski \\ (traducción de Luisa Puig)
}

\begin{abstract}
Este artículo es una reflexión que parte de una teoría sociocrítica y propone articular el estudio sobre textos literarios escritos por mujeres en una combinatoria teórica tridimensional: ciertos postulados teóricos de Bajtín —en particular lo concerniente al estudio dialógico del discurso-, las investigaciones en sociocrítica sobre la heterogeneidad constitutiva de la realidad textual y algunas orientaciones de la crítica feminista, en especial las que buscan reformular la cultura desde una perspectiva feminista.
\end{abstract}

This article takes as a point of departure a sociocritique theory, and proposes the study on literary texts written by women as a combination of three theoretical dimensions: the combination of some postulates from Bakhtin, particularly those concerning his dialogical theory of discourse; with research on sociocritique about the constitutive diversity of textual reality, and with some orientations from the feminist critique, especially those that seek to reformulate culture in a feminist perspective. 



\author{
M.-Pierrette Malcuzynski \\ (traducción de Luisa Puig)
}

\title{
Yo no es un O/otro
}

\section{Nota preliminar}

Esta intervención retoma los puntos esenciales de una reflexión sobre teoría sociocrítica feminista, reflexión que forma parte de un proyecto de investigación en curso sobre el surgimiento y el desarrollo de las formaciones discursivas en los textos literarios hispánicos (españoles e hispanoamericanos) escritos por mujeres. Se trata, pues, de aseveraciones que se inspiran en una serie de intervenciones y de conferencias, así como de publicaciones anteriores o actualmente en prensa, la mayoría en español. ${ }^{1}$ El presente trabajo busca desarrollar ciertos aspectos teóricos de la aportación del dialogismo bajtiniano en lo que toca a la problemática en su conjunto. Precisemos, por otra parte, que si la sociocrítica es una disciplina que no puede negar su origen bajtiniano en lo que concierne a muchos de sus postulados, ningún investigador o investigadora que, por lo demás, se considere "sociocrítico" o que se reclame partidario de la sociocrítica, sea cual fuere su orientación,

${ }^{1}$ Para una selección representativa de esta investigación, véanse mis artículos de 1993, 1994 y 1995, citados en la bibliografía, a los que habrá que añadir un estudio monográfico, actualmente en preparación, sobre las Memorias de Leonor López de Córdoba (principios del siglo xv) (1997, para el texto de estas Memorias). 
ha desarrollado un discurso que tome en cuenta, de manera íntegra, los textos escritos por mujeres como objeto de estudio específico. ${ }^{2}$ Es ésta una situación, por lo menos curiosa, viniendo de una disciplina en vigor desde hace cerca de 25 años y que se relaciona, entre otros temas, con el análisis del discurso y de los discursos. Habremos, pues, partido de cero en la articulación de una teoría sociocrítica pertinente con el problema particular que presenta el examen de los objetos socioculturales literarios, cuando el sujeto que habla y escribe en el texto es una mujer.

Al mismo tiempo que busca articularla, la hipótesis de una teoría sociocrítica feminista se sitúa en una combinatoria de intersecciones tridimensionales de la siguiente manera:

1. Algunos de los postulados teóricos de los escritos de Bajtín / Voloshinov, en particular los que remiten a la coyuntura comunicacional del trabajo dialógico sobre el discurso.

2. Las modalidades metodológicas sociocríticas de base, a propósito del trabajo sobre la heterogeneidad constitutiva de la realidad textual. Esto significa examinar no sólo la manera como se dice lo que se dice, sino también leer los "blancos" de un texto, "escuchar" y descifrar sus silencios, descodificar los no dichos y, lo que no es lo mismo, los indecibles, los "inconfesables". Es decir, desarrollar, a nivel del texto, todo lo que Michel Foucault definía en términos de lo que puede ser dicho "aquî" y no "allá", y viceversa.

3. Ciertas orientaciones de la crítica feminista de los años ochenta ${ }^{3}$ que buscan reformular la cultura desde una perspectiva feminista, distinguiéndose de las proposiciones que giran alrededor de una "feminización de la cultura", cuyas premisas podríamos resumir como una pura y simple inversión de la

2 De hecho, ciertos sociocríticos piensan que desarrollar un discurso teórico feminista desde el "interior" de la sociocrítica no es realmente "necesario".

${ }^{3}$ Esta perspectiva se basa, en parte, en las proposiciones de Adrienne Rich (1979) como la "Re-visión" o "reescritura de la cultura patriarcal". 
premisa lacaniana "yo es un Otro" en "ese otro soy yo". A primera vista, reformular la cultura en tal perspectiva consiste en reemplazar el paradigma "la mujer como el otro" por "la mujer y el otro", "el otro" entendido en el sentido amplio, ontológico y cognitivo del término. Esta reorientación de base permite a Myriam Díaz-Diocaretz $(1989,126)$ postular un acercamiento "situado en el cruce entre una crítica feminista que considera al lenguaje como un fenómeno no determinista y la teoría de la comunicación de Mijaíl Bajtín" (la traducción del inglés es mía; 1989, 126). ${ }^{4}$ Insuficiente me parece, en cambio, la continuación de esta proposición — que sin embargo no deja de ser una de las más sugestivas en la teoría crítica feminista contemporánea-, la cual implica el estudio de " 'ese otro' en el discurso, como también de las 'voces', las formas, las correlaciones y los contextos de ese 'otro', cuando el sujeto que habla y escribe es una mujer” (Malcuzynski 1991, 136).

Lo esencial del trabajo por hacer se sitúa, en mi opinión, a nivel de la conjunción y del nuevo paradigma. Así, a nivel teórico, es posible vislumbrar la construcción de una sociocrítica feminista, en cierta forma como una cuarta dimensión de la problemática de conjunto, que permite que los tres ejes de la combinatoria interactúen recíprocamente. Pero, tengamos cuidado: no se trata simplemente de poner en relación y de confrontar elementos contiguos provenientes de prácticas críticas que, en suma, son bastante diferentes los unos de los otros. Para valorizar la dinámica interactiva de sus funciones respectivas, reiteremos, con Bajtín, el hecho de que incluso la estética (literaria) "necesita forzosamente de definiciones recíprocas con los otros campos, en la unidad de la cultura" ("Le problème

${ }^{4}$ Consultar también de M. Díaz-Diocaretz, "El sociotexto: el entimema y la matriherencia en los textos de mujeres" (Malcuzynski 1991, 129-144) y "La palabra no olvida de dónde vino". Observemos que M. Díaz-Diocaretz es una de las raras críticas feministas que, en vez de aplicar mecánicamente tal o cual modalidad de origen bajtiniano al texto escrito por la mujer, aprovecha los postulados teóricos de Bajtín, retrabajándolos en una perspectiva específicamente feminista. 
du contenu, du matériau et de la forme dans l'oeuvre littéraire", Esthétique et théorie du roman, 26). Se trata sobre todo de acentuar los procesos dialógicos de estas interacciones, con el fin de mitigar los riesgos de llevar a cabo, de nuevo y siempre, una polarización binaria velada: los co(n)textos ${ }^{5}$ de la mujer, por una parte, y los del "otro", por la otra. A nivel de la práctica crítica, esto significa, en cierta forma, asir cognitivamente esta intersección vital y extraer los modos de articulación de la preeminencia de lo interdiscursivo sobre el discurso, para dar cuenta de las prerrogativas ontológicas y sociales de los diversos empleos de los discursos, ellos mismos provenientes de diversas clases y categorías. Para lograr lo anterior, propuse, hace algunos años, un concepto operador que denominé monitoring, ${ }^{6}$ el cual busca precisar y profundizar, en una perspectiva sociocrítica, el trabajo (creador, crítico) del sujeto, trabajo que Régine Robin y Marc Angenot (1985) llamaron "la escucha del discurso social", pero que no considera al sujeto más que en su acepción neutra, agenérica (genérica en el sentido inglés del término gendered). Por monitoring se busca, en primer lugar, interpelar la polifonía discursiva de manera comprensiva, trabajando, por una parte, las interacciones dialógicas intersubjetivas, las que definen las relaciones entre diversos sujetos, los cuales, a través de su práctica (textual), manipulan materiales discursivos igualmente diversos y no sólo lingüísticos y, por la otra, las relaciones dialécticas en-

\footnotetext{
5 Consultar Robin (1993) sobre la diferencia entre "co-texto" y "contexto".

${ }^{6}$ Cf. Malcuzynski 1989 y, en particular en 1991 [dir.] ("El monitoring: hacia una semiótica social comparada", 145-174); por otra parte, se encontrarán los primeros esbozos de una reorientación feminista del concepto en los artículos de 1993 y 1995. En lo que concierne al término mismo, inicialmente me inspiré en el título de un programa de la Televisión Nacional Polaca, Monitor - un oneman show sobre la política nacional e internacional-, difundido en los años sesenta. Observemos, en fin, que mi concepción de monitoring no tiene absolutamente nada que ver con los trabajos provenientes de ciertos campos de la (psico) lingüística, que utilizan el mismo término para designar ciertos mecanismos de la producción del lenguaje en el individuo.
} 
tre los sujetos y sus objetos. Distinguiendo, por otro lado, entre valor de cambio y valor de uso, el monitoring requiere de una teoría de la mediación que remita no sólo a la circulación de los discursos, sino también a su producción y su materialización, en el seno de un estado de sociedad dado, es decir al sujeto productor.

El punto de partida conceptual es la noción bajtiniana de umbral. Ahora bien, esta noción no designa una especie de no man's-land, un constucto in abstracto, un topos digamos neutro o indeterminado. En mi lectura de Bajtín, estar en el umbral significa "situarse en la frontera", en un lugar de convergencias múltiples y multidimensionales. El objetivo es el de desdoblar el mapa (me atrevería casi a decir topográfico) de ese complejo sociointerdiscursivo que llamamos texto, abrir un espacio distintivo de análisis textual que interpela una serie de relaciones concretas, materiales de producción, de orden transsemiótico e interdisciplinario de facto, entre una práctica socioideológica definida y la práctica cultural, entre otras literaria. Más que un trabajo sobre los problemas (eternos) de la representación, damos cuenta de una reevaluación de la constitución del sujeto y del discurso relativo a la construcción de la identidad sociocultural. Por consiguiente, hablamos en efecto de una lectura que, fundada en la premisa bajtiniana de la heterogeneidad social de la circulación del lenguaje y de la comunicación, restituye, al análisis del sujeto, una problemática cognitiva en el seno de una economía epistémica de "responsabilidad". En lo que concierne a la práctica feminista, se trata sobre todo de resistir a las configuraciones constituidas por toda clase de oposiciones maniqueístas que desembocan en la exclusión o, en todo caso, en la relegación del sujeto femenino (y/o feminista, poco importa), aislándolo en un proceso de autorrepresentación periférico; todo lo relacionado con lo que antes se llamaba, de acuerdo con Deleuze y Guattari y en otro contexto, los procesos de desterritorialización. Resis- 
tencia también a las síntesis metafísicas agenéricas que engendran discursos "antropófagos" y que manipulan factores de neutralización. La voz supuestamente neutra producto de una única verdad impersonal y monolítica es, en mi opinión, un sofisma absurdo. Hablar nunca es neutro; nos lo recuerda el título de la obra de Luce Irigaray.

Detengámonos un instante en este fundamento epistemológico y recordemos que una teoría bajtiniana del discurso afirma que no sólo se trata de lo que acontece "al interior" de nuestra propia conciencia, sino en la frontera de la conciencia de otro sujeto cabal, completo, precisamente en el umbral. Para Bajtín, el más alto grado de socialidad estriba en el hecho de que cada experiencia interna, cada sujeto, termina por toparse con otro. Toda la "ontología del yo" en el sentido bajtiniano se dialogiza, en primera instancia, en esta frontera, y no puede realizarse más que en este lugar de encuentro lleno de tensiones. El sujeto siempre es el producto de su interacción con otros sujetos. "El acontecimiento en la vida del texto, su ser auténtico, siempre tiene lugar en las fronteras de dos conciencias, de dos sujetos" ("Le problème du texte", en 1984, 315). Y también:

El acto verdaderamente creador (y, en realidad, todo acto) evoluciona en las fronteras (en las fronteras de los valores) del mundo estético, de la realidad de lo dado (la realidad de lo dado es una realidad estética), en las fronteras del cuerpo, en las fronteras del alma, evoluciona en el espíritu; en cuanto al espíritu, no existe todavía; para el espíritu, todo está aún por venir, y lo que ya es, para él ya fue ("L'auteur et le héros", 1984, 209).

El otro (interlocutor/discurso) siempre es el otro del otro (interlocutor/discurso). Esto permitió a Jacqueline Authiez-Revuz (1982) afirmar que el otro del Otro (con O mayúscula, inconsciente) no existe en una concepción bajtiniana de la problemáti- 
ca del sujeto. De manera que el corte interior de origen psicoanalítico - aquel que hace que la teoría del sujeto se deslice en una "alteridad" del yo cuya explosión interna se interpreta, a partir de entonces, como el origen formativo de un discurso plural - es una fórmula que permanece ajena al pensamiento bajtiniano. De igual forma, no confundamos las relaciones de "ambivalencia", de "paradoja", de "ambigüedad", etc., que mantienen entre ellos los jirones fragmentarios del texto postestructuralista / modernista, con el diálogo social bajtiniano. Olvidaríamos de golpe al sujeto productor. ${ }^{7}$ No nos cansaremos nunca de remitir explícitamente al problema que presentan estas tendencias en la teoría y la crítica literarias, en vigor todavía hasta la fecha, aglutinadas alrededor de los conceptos que acabo de mencionar, la mayor parte de los cuales fueron, por lo demás, engendrados en las primeras lecturas e interpretaciones, bastante raras, de Julia Kristeva de los escritos bajtinianos, en particular del dialogismo, de lo polifónico y de la carnavalización.

Seamos precisos: revisar el monologismo idealista hegeliano a través de un prisma psicoanalítico ciertamente no podía más que conducir a una, digamos, "nueva" dialéctica que vendría a ponderar un sincretismo freudiano marxista triunfante. Habrá, en cambio, atestiguado la entronización de este doble O/otro supremo (por lo demás, siempre "suyo", nunca "suya") y que, sin embargo, logró canonizar Lacan, como un magistral Nietzsche contemporáneo, pero con las patas al aire, si se me permite la expresión (o, según sea el punto de vista, con la nariz por tierra). Hago referencia a esta óptica perfectamente ahistórica que se precisa a partir de una ruptura interna, paraesquizoide, fundada en una problemática del subconsciente y transmitida por una pseudometafísica psicoanalizante: mirada

\footnotetext{
${ }^{7}$ Es decir, quién escribe, para quién y a quién se dirige uno, desde qué espacio cronotópico (geopolítico, social, cultural, etc.) y cuándo, cómo y, sobre todo, por qué.
} 
atemorizada del [Sobre-]Mí en tanto que reflejo (post-?)estructural de un deseo que no puede o no pudo obtener su objeto. Y así nació esta confusión masiva entre producción cultural, por una parte, y productividad de los sistemas simbólicos, por la otra, ahí en donde, precisamente, a través de aquello que no tengo ningún escrúpulo en llamar un lapsus monumental, Kristeva (1970) pudo afirmar que el Sujeto ${ }^{8}$ con $S$ mayúscula está (bien) muerto. Sin embargo, la cultura y sus objetos no pueden considerarse objetos de intercambio, transferidos, por así decirlo, o "diferidos" de un sistema de referencia a otro por un sí o por un no. En lugar de fusión, de exclusión o de sustitución —en definitiva, de la deposición sistemática, velada o no, de un discurso por otro, es decir, de una recanonización-, el dialogismo implica una práctica de acercamientos y de tomas de contacto entre fenómenos usualmente aislados los unos de los otros. Evidentemente, no se trata de una simple acumulación de saberes en la que la erudición consistiría en recolectar discursos heterogéneos y amontonarlos, unos encima de otros, para constatar la evidencia de la "diferencia" y de los "diferendos" en el seno de cualquier pluralidad, a reserva de olvidar o de borrar esta evidencia lo más rápidamente posible. Hablamos, más bien, de una práctica cognitiva y ética que interpela a la heterogeneidad de lo "plural" de manera que no se diluyan en ella las propiedades o las particularidades que lo constituyen.

Me opongo a las interpretaciones anaxiológicas y ahistóricas que hacen de la relación dialógica, en el sentido bajtiniano del término, una amalgama sincrética resultante, a la vez, de Arthur Rimbaud, de Mijaíl Bajtín y de Jacques Lacan. En otras palabras, a nivel del discurso, "Yo [no] es un O/otro": si yo no puedo ser yo sin aceptar la relación interactiva y participativa contigo/interlocutor —el otro sujeto/discurso íntegro-

\footnotetext{
${ }^{8}$ Por lo demás, muy diferente del Autor (barthesiano).
} 
pero que no es yo, esto no quiere decir que yo no sea yo, sujeto/discurso también íntegro, y que no podría confundirse con "un otro" ni con "el Otro del otro". "Yo también soy", dice Bajtín. ${ }^{9}$ Se trata, antes que nada, de desenmascarar las diversas estrategias que, todavía y siempre, incluso en el seno de las más radicales teorías críticas contemporáneas, hacen recaer la "voz" de la mujer en la trampa tendida por las múltiples configuraciones de polarización binaria, característica del fetichismo de la lingüística. Ahora bien, en la actualidad ya no basta con dar cuenta de la evidencia de que el lenguaje es, en sí mismo, portador de la institución patriarcal, ni de asumir, incluso de manera crítica, el discurso "minoritario" al que la ideología (cultural) dominante — es decir, el discurso social— reduce el sujeto femenino que escribe en el texto. En definitiva, el problema más apremiante, observaba hace poco Toril Moi, es cómo evitar el empleo de concepciones machistas — canónicas- a propósito de la estética, de la historia y de la tradición, cuando el sujeto que habla y escribe es una mujer. Y esto tiene precisamente que ver con un problema fundamental de ética.

Me parece mucho más urgente trabajar la formación y la estructuración del "o" de los discursos a partir de los que se articulan las formas de representación. Desde este ángulo, más importantes que la línea divisoria a partir de la cual o sobre la cual se (re)constituye un imaginario canónico, sean cuales fueren justamente sus formas de representación, ${ }^{10}$ son las es-

9 "I, too, exist" (Bajtín, Philosophy, 9).

${ }^{10}$ En lo que concierne a los estudios literarios, pienso en la consigna que consiste en afirmar que la tarea feminista por excelencia es la de reescribir el canon (cf. "Las formas y funciones de una teoría crítica feminista. Feminismo dialógico" de Iris M. Zavala). Es éste un argumento peligroso, sobre todo para una aproximación que se reclama bajtiniana, y que corre el riesgo de patinarse en todos los sentidos. En particular, vería en ello el peligro de unirse, a pesar de nosotros mismos, a esas nuevas orientaciones radicales de recuperación ultraconservadoras de lo que podría llamarse "el aparato o los aparatos canónicos" sobre los que la ideología disimula mal sus premisas cripto-fundamentalistas, ahí donde Harold Bloom pudo decir que sin el Canon, con C mayúscula, se deja de pensar ("Without the Canon, we cease to think", 41). Señalemos claramente que los propósitos sustentados en los cuatro vo- 
trategias socioideológicas que negocian y potencializan una prerrogativa que he llamado diferencial. Me inspiro aquí en lo que en matemáticas significa un diferencial — que remite a la diferencia creada por el aumento infinitamente pequeño de una función, correspondiendo al aumento infinitamente pequeño de una variable - y, sobre todo, de lo que la noción nos aporta a partir de su significación en el terreno de la mecánica. Es decir, un mecanismo que entrelaza tres móviles, imponiendo las condiciones para que cada una de sus velocidades simultáneas sea proporcional a la suma o a la diferencia de las otras dos. ${ }^{11}$ Estas dos definiciones de "diferencial" nos acercan, respectivamente, del concepto del grado cero del dialogismo y de la perspectiva desjerarquizante que nos ofrecen las teorías bajtinianas sobre la polifonía literaria. Y sugerí, hace ya algunos años, abordar ese grado cero como el punto o uno de los puntos de partida metodológicos de la práctica sociocrítica (cf. Malcuzynski, 1989, 1990, 1992).

Según lo que se puede deducir de las sugerencias bajtinianas, el concepto del grado cero designa una especie de cuarta dimensión en la dinámica tripartita del dialogismo, espacio o topos en el que Bajtín introduce "el punto de vista de un 'tercero' que no participa formalmente en el diálogo pero que lo comprende" (Bakhtin 1978, 335). Es justo a nivel de esta reflexión donde se materializa el abismo que, como yo lo entiendo y en una perspectiva cognitiva, se perfila entre "interpretar" y "comprender" en el sentido bajtiniano del término. Es decir, entre, por una parte, interpretar el mundo, los objetos

lúmenes ya publicados, de los cinco o seis anunciados, sobre una historia feminista de la literatura española (Díaz-Diocaretz y Zavala, 1993 y Zavala, 1995, 1996, 1997), encierran numerosas aportaciones innovadoras que, a partir de ahora, habrá que tomar en cuenta, tanto en lo que concierne a la historia literaria española como a la crítica feminista en general. Ciertas proposiciones, en cambio, como la mencionada arriba, suscitan problemas muy delicados cuyas consecuencias teóricas no es posible ignorar.

11 Ver Malcuzynski (1999) para una discusión sobre el "sonido diferencial" en acústica musical. 
socioculturales, etc., y considerar la diferencia (diferencia tomada aquí en tanto que concepto axiológico) como una entidad cerrada, objetivada, siempre plural ciertamente, pero abordada y cercada en su aislamiento en tanto que "otra extranjera" y, por la otra, comprender la pluralidad en tanto que diversidad; comprender el mundo en y desde la diversidad, a partir de las relaciones y de las interrelaciones entre las diferencias que constituyen el mundo en el sentido de totalidad en cualquier momento dado de la historia. En el seno de una teoría sociocrítica de la interdiscursividad, avanzaré la idea de que, a partir del grado cero dialógico de Bajtín, las modalidades estratégicas del diferencial feminista articulan una secularización del discurso.

Propongo, por consiguiente, trabajar la problemática del sujeto no de manera "reactiva" frente a la axiología canónica en vigor en tal o cual estado de sociedad dado, y las formas patriarcales de división genérica a partir de un bloque hegemónico, sino desde el interior de los procesos interdiscursivos manifiestos por y en el texto, ahí donde hablar de lo intertextual no tiene precisamente ningún sentido si no se sitúa en relación con las diversas prácticas sociodiscursivas que sostienen y atraviesan el texto. Para Bajtín, "el texto no es un objeto, por lo que es imposible eliminar de él o neutralizar en él la segunda conciencia, la conciencia del que cobra conocimiento del texto" ("Le problème du texte", en 1984, 315). Tal como aludíamos más arriba, no podríamos confundir la relación dialógica entre dos o varias conciencias, la cual permite al acontecimiento vivo, que es la idea, desarrollarse, con la relación dialéctica que caracteriza la unión de la idea y de la palabra (Bakhtine 1970, 129), lo que Michel Pêcheux llamaba, en otro contexto, "el imaginario lingüístico".

Probemos el argumento siguiente: aquello que se ha traducido en los trabajos de Bajtín en términos de lo "dado" y de lo "creado" remite muy concretamente a una serie de interrela- 
ciones cognitivas que no son para nada binarias. Muy por el contrario y al igual que la relación dialógica (cf. Bakhtine/Volochinov 1977), se trata de relaciones fundamentalmente tripartitas que incluyen un "posé"” ("posited" en la traducción inglesa; "planteado" de acuerdo con Tatiana Bubnova, la traductora al español de Bajtín). Yo prefiero hablar en términos de "proyectado", en el sentido de "proyección" de horizontes ideológicos, epistemológicos. Bajtín señalaba que hay dos factores que entran en juego en la determinación de un texto (lo "creado") y que "conforman un enunciado: el designio (la intención) y la ejecución de ese designio" ("Le problème du texte", en 1984, 312), ${ }^{12}$ ahí donde se insertará el designio a la categoría de lo "proyectado" y su ejecución a la de lo "creado", y que hace que lo "proyectado" pertenezca siempre a lo "creado". Lo "creado" no puede actualizarse en la sola relación que mantiene con lo "dado". De hecho, lo "creado" no es entonces posible, no es concebible más que en la frontera de lo "dado" y de lo "proyectado". En lo que concierne a una reformulación de la cultura en una perspectiva feminista, falta por hacerse todo un trabajo teórico a nivel de esta distinción incuestionable entre "discurso" y "texto" en el seno de la relación que las nociones bajtinianas de ética y de estética mantienen entre sí, cuando el género (gender) del sujeto es considerado como una categoría de análisis específica.

Llegamos al corazón de la problemática de la producción del sentido. El sentido siempre es una variable coyuntural pero que no podríamos considerar sinónima de lo polisémico. No confundamos esta "comprensión" (en el sentido estricto del término) de la noción de "sentido" con un punto de vista objetivado, vago, transhistórico y por el cual se asimilaría a

* Término en francés (nota de la traductora).

${ }^{12}$ La cita continúa: "Interrelación dinámica de esos dos factores, su lucha que imprime el carácter al texto. Un desacuerdo entre esos factores puede decir mucho. El lapsus escrito, oral, según Freud (expresión del inconsciente). La modificación del designio en curso de ejecución. La no realización de la intención fónica”. 
una "Verdad" simbólica de significación, impersonal a nivel paradigmático, es decir, "universal" en una acepción, digamos, neutra de su formulación.

Entendámonos bien: el sentido no es una entidad sino un proceso, un proceso por lo demás dialógico y no dialéctico. Es nuevamente Bajtín quien da cuenta de esta afirmación. "El sentido siempre responde a una pregunta. Lo que no responde a nada nos parece insensato, se desliga del diálogo", explica, agregando "que no hay un 'sentido en sí mismo'. El sentido no existe más que para otro sentido, con el que existe conjuntamente. El sentido no existe solo (solitario)" ("Les carnets 1970-71", en 1984, 366). Es ésta, sin duda, una distinción clara y precisa que permite establecer que lo que la significación sería a la dialéctica es lo que el sentido es al diálogo. Así, el sentido no es una categoría cuantificable lingüísticamente, ahí donde la palabra, subraya Bajtín, "revela sus significaciones sólo en contexto". Tal como la ideología, el sentido sólo es identificable, pero no porque el discurso no tenga frontera, como si fuera un espacio que, por definición, transgrediera todos los límites — postulado apreciado por los posmodernos - sino porque el discurso es, en sí mismo, una frontera. El matiz es sutil, es cierto, pero fundamental: el discurso es una frontera porque se trata de una instancia dialogizada en la que múltiples conciencias, provenientes de diversos horizontes, confluyen para constituir una coyuntura determinada. En el seno de esta coyuntura, sociopolifónica y epistemológica, el sujeto siempre toma posición.

La noción de imaginario canónico que hace un momento adelantamos exige, evidentemente, desarrollos que no es posible discutir aquí, ${ }^{13}$ pero señalemos que en sus aspectos asimilados o rechazados, de acuerdo con el estatus y la función del

13 Todo un trabajo taxonómico falta por hacer para desembrollar los diversos usos de la noción de imaginario, desde su empleo en el sentido técnico lacaniano, a las diversas acepciones de "el imaginario [de lo] social", pasando por la noción bajtiniana [Voloshinov] de subterráneo político y aquella, demasiado vaga, de inconsciente político de Fredric Jameson, entre tantos otros. 
sujeto en el seno de la polifonía discursiva, el contenido canónico del imaginario siempre forma parte integrante del texto. Podríamos decir que lo canónico es lo "no decible" de toda formación imaginaria y, como tal, de cierta manera se identifica con lo que sería el hábito de su discurso. En consecuencia podemos argumentar que cuando la contingencia genérica es comprendida como una categoría de análisis propiamente dicha de la constitución del sujeto, la institución del patriarcado aparece entonces como un hábito con ramificaciones múltiples. Podemos hacer visible este hábito patriarcal explicitando las dos etapas cognitivas que el sujeto atraviesa cuando es una mujer la que habla y escribe, en particular cuando ese sujeto estructura su identidad sociocultural a partir de un discurso específicamente feminista: primeramente $-\mathrm{y}$ contrariamente a lo que nos enseñaron Michel Pêcheux, ${ }^{14}$ Louis Althusser, in-

14 Remitimos, evidentemente, a la teoría de los "dos olvidos" de Michel Pêcheux (1975 a y b), a la que habremos hecho varias veces alusión en el curso de esta intervención. Recordemos específicamente la argumentación de Pêcheux: el olvido $\mathrm{n}^{\circ} 1$ devuelve la constitución del discurso a una zona de naturaleza inconsciente, inaccesible al sujeto: en otras palabras, por analogía a la relación que establecía Lacan entre el yo y el Otro, con $O$ mayúscula, el sujeto "olvida", reprime el sentido que se forma en procesos que le son exteriores. Por otra parte, el olvido $\mathrm{n}^{\circ} 2$ que, de manera no simétrica, sitúa los procesos de enunciación en la zona del preconsciente, al mismo tiempo que remite a un problema de identificación imaginaria en el que el otro, con $o$ minúscula, se vuelve otro yo. Así se postulaba la hipótesis de la ilusión discursiva: “ilusión”, en primer lugar, por parte del sujeto, de estar en el origen del sentido bajo la forma de la recaptura por [él] de un sentido preexistente universal y, en consecuencia, de producir efectos de ocultación ("todo aquello que le habría sido posible decir al sujeto" pero que no dijo o de "todo aquello que se opone a lo que dijo el sujeto"). Impresión "de realidad del pensamiento", en consecuencia, "ahí donde reside la 'libertad' —que Pêcheux pone entre comillas — del sujeto hablante ('sé lo que digo', 'sé de qué hablo')". La diferencia, la desigualdad entre los dos olvidos ("lo no afirmado precede y domina lo afirmado") corresponde a una relación de dominación: la represión que caracteriza el olvido $\mathrm{n}^{\mathrm{o}} 1$ regula, a fin de cuentas, la relación entre lo dicho y lo no dicho en el olvido $n^{\circ} 2$, donde se estructura la secuencia discursiva". Y Pêcheux nos permite recordar, citando a Lacan, que todo discurso es ocultación del inconsciente. "El efecto de la forma-sujeto del discurso es, pues, en primer lugar, el de ocultar el objeto del [...] olvido $\mathrm{n}^{\mathrm{o}} 1$, por el sesgo del funcionamiento del olvido $n^{\circ} 2$ ", agrega éste, de manera que "de reformulación-paráfrasis que caracteriza una formación discursiva dada aparece como el lugar de constitución [de un imaginario lingüístico]". 
cluso Fredric Jameson (el de El inconsciente político) y tantos otros que fundaban sus reflexiones en una episteme de la amnesia, en cuya base se encuentran las teorías neofreudianas contemporáneas-, sin olvidar sobre todo que las "voces" que articulan la hegemonía patriarcal desde el interior del discurso son, ellas mismas, las que encuadran, dirigen y legitiman, desde el exterior, las estructuras discursivas oprimidas, en ocasiones llamadas "minoritarias" frente a las consideradas "mayoritarias" del discurso social. Y en una segunda etapa, tal vez aún más importante, sin olvidar tampoco el hecho de que, al aceptar las formas de su propia contradicción interna, el sujeto tiende a marginalizarse y a proyectar al exterior ese constructo binario, esencial para esta misma axiología hegemónica, cuya ideología constituye el fundamento de nuestra epistemología occidental, y que la coyuntura posmoderna expresa en términos de "la ambivalencia", de "la paradoja", de "la ambigüedad", etcétera. ${ }^{15}$ Antes que nada y sobre todo, por teoría sociocrítica feminista se afirma una práctica irreductible a las modalidades monolíticas que confronta; modalidades que, en nuestra época llamada "pluralista", tal vez más que en otras, pero en ningún caso exclusivas de ésta, fundan su eficacia apropiándose del diferencial como suyo y, al mismo tiempo, fijan o "canonizan" los contenidos periféricos en tanto que orden legítimo y aceptado como tal, en la óptica de esta misma hegemonía. ${ }^{16}$ Frente a este aparente callejón sin salida, es desde ahora necesario cambiar de pie, recobrar una focalización crítica que extraiga las estrategias a las que recurre el sujeto cuando estructura su socialidad, para poder plantear el problema de la tensión inherente de la heterogeneidad y lograr re-

15 De igual forma, los conceptos de "indeterminación" y de "diferencia" (cf. Derrida) no son sinónimos de "diferencia" y aún menos de "diferencial"; tampoco sería posible confundir el de "diseminación" con el de "diversidad polifónica".

16 Hace ya tiempo, Jean-François Lyotard calificaba este mecanismo de reapropiación posmoderna como paralogía. 
configurar las relaciones entre lo comunicacional y las formas de las significaciones. Más que buscar inventarse un, digamos, "nuevo" lenguaje, remito a una política sociocrítica que lleve a cabo una hermenéutica responsable de mediación cultural; es decir, que descolonice, por decirlo así, la problemática genérica impuesta por el patriarcado, y desmarginalice al sujeto femenino sin por ello neutralizar su toma de posición.

En lo que concierne a los procesos de la puesta en texto, esta toma de posición se enuncia dialógicamente para recubrir una prerrogativa discursiva que surge de la conjugación entre lo que llamé en otra parte, lo "ya ahí" y "lo que todavía no está". Entendemos esta conjugación no como un cierto voluntarismo dogmático sino como una manera de repensar el mundo; se trata menos de atacar o de negar, que de concebir horizontes diferenciales - restituir un poco de utopía, sobre todo ahí donde parece ya no haber, nunca ha hecho daño, que yo sepa- Hago alusión a lo que quisiera ser la ilustración del diferencial como función del "cambio". Ahora bien, todos sabemos que el cambio no puede ser abordado en términos de una división entre el poder y el saber, sino en una óptica que pone en relación específicamente el ejercicio del poder y la adquisición del saber. Y esto significa confrontar ciertas opiniones que afirman que el cambio no es visible más que a posteriori, a título de hecho realizado. Ahora bien, si es posible afirmar que el cambio no puede asumirse más que en una puesta en perspectiva retroactiva, creo, sin embargo, que no sólo se debe buscar delimitar los factores que potencializan el cambio, sino igualmente que el cambio en sí mismo puede identificarse como tal en lo inmediato de su manifestación. Es sólo a ese nivel ético de "responsabilidad" cognitiva donde puede utilizarse una re-territorialización. Me parece entonces posible concebir una semiosis diferencial en la que los campos del saber y de la experiencia no se confundan recíprocamente, según los principios del recorte de la semiología tradi- 
cional. Y no olvidemos que las formas manifiestas de ese recorte - hábito negativo semiológico del fetichismo lingüístico- proyectan una dicotomía maniqueísta que, en sus formas binarias de representación, sirven de fundamento y de razón de ser al poder que la noción misma de canon ejerce sobre la legitimación de la cultura, e igualmente sobre la formación y la circulación de los discursos, así como sobre sus diversas interpretaciones.

Bajtín escribía en su primera publicación conocida (Art et responsabilité, 1919) ${ }^{17}$ que "el arte y la vida no son la misma cosa, pero deben convertirse en mí en algo unitario, al interior de la unidad de mi responsabilidad", fundamento ético retomado a propósito del acto (cf. Bakhtin 1993) y que elaboraría ulteriormente en diversas direcciones estéticas, a medida que fue desarrollando su reflexión sobre la cultura literaria. Para volver a dar vigor a esta constatación propongo, junto con Walter Benjamin - ese contemporáneo de Bajtín cuyo pensamiento crítico aún queda por examinar de manera sistemática en relación con el de Bajtín—, ${ }^{18}$ considerar la literatura como un organon de la historia (cf. 1979-1983). ${ }^{19}$ Sin perder de vista lo que "organon" significaba para Aristóteles en sus tratados de Lógica, la sugerencia nos permite abordar la literatura, en primer lugar, como un sistema de demostraciones que ayudan a organizar un determinado razonamiento del conocimiento. Es en este sentido preciso como Francis Bacon empleaba el tér-

${ }^{17}$ Este artículo ha sido omitido de la traducción francesa de Estetika slovesnogo tvortchestva; figura sin embargo en versión española (trad. de Tatiana Bubnova) al principio de Estética de la creación verbal, México, Siglo XXI, 1982.

${ }^{18}$ Recordemos al respecto el trabajo de Terry Eagleton (143-172), relativamente desconocido de la crítica francófona.

${ }^{19}$ Sería efectivamente interesante, por ejemplo, examinar de manera crítica, en una perspectiva comparativa, ciertas propuestas de Benjamin sobre la historia literaria con las de Bajtín, entre otros en sus artículos "Les études littéraires aujourd'hui" (1970), "Les carnets 1970-71" y "Remarques sur l'épistémologie des sciences humaines" (cf. 1984, 338-393). 
mino latino, en su Novum organum en lo concerniente al saber y al razonamiento científico. Tal aproximación a la pragmática de los saberes difiere radicalmente de lo que sugiere la noción de canon, por lo demás muy bien resumida por Leibniz: "Llamo cánones las fórmulas generales que antes que nada y sobre todo nos ofrecen lo que pedimos". Queda por precisar, desde luego, quién pide qué y en qué contexto.

Esta diferencia se vuelve aún más clara si nos referimos al canon musical y al advenimiento de las técnicas contrapuntistas de composición en la época medieval, cuando el término de "canon" en ese sentido se asimila al de repetición, de manera análoga a una "norma" o modelo a seguir. El canon musical consiste efectivamente en la rigurosa imitación de un canto principal dominante, llamado más o menos el antecedente, por una segunda o por varias voces, la o las consecuentes, que entran sucesivamente las unas después de las otras al unísono o a intervalos regulares superiores o inferiores. Es importante subrayar aquí que la melodía principal es la que da la regla y el paso a todas las otras que deben imitarla, nota a nota. A este nivel de asociaciones teóricas musicales, pienso igualmente en este otro aspecto del desarrollo del contrapunto, que llevará a constituir técnicamente la polifonía: es decir, una multiplicidad de voces que cantan simultáneamente diferentes melodías y cuyo principio de equipolencia retuvo Bajtín, mucho más escrupulosamente, por lo demás, que lo que parece a primera vista, cuando concebía y definía el discurso polifónico a partir de una lectura de Dostoievski, y lo oponía a un discurso monológico literario, de la misma manera como la polifonía se opone a la monodia en música. Es importante subrayar aquí que la aproximación bajtiniana consiste menos en fabricar un paradigma literatura/música sobre el que él mismo señala las dificultades, que en determinar lo que la oposición monodia/polifonía en la historia de la música puede enseñarnos, al establecer una relación de la función de esta oposición en lo concer- 
niente a la historia de la literatura y, en particular, de la novela. ${ }^{20}$ Esta aportación teórica es la que buscamos retomar en una óptica específicamente feminista.

El primer tratado de música que hace una clara referencia a la posibilidad de componer canciones para diversas partes data de la primera mitad del siglo viI. Habrá de todas maneras que esperar el fin del siglo ix para que aparezca una definición explicativa y teórica de las técnicas de composición polifónicas. Así, fundada en la doctrina pitagórica de las consonancias, la técnica de la diafonía consistía en la concordancia simultánea de dos voces o grupos de voces que cantaban horizontalmente la misma melodía en una textura diferente. La vox principalis designaba la melodía principal, mientras que la vox organalis era la que reproducía, simultáneamente y a un intervalo determinado, la melodía a título de acompañamiento de la voz principal. Dicho de otro modo, la palabra latina organon, organum designaba, en el origen, una parte cantada a una cuarta o a una quinta de intervalo en tanto que acompañamiento de la melodía principal. Es aquí donde, desde un punto de vista teórico, todo un mundo musical de diferencia puede concebirse, toda una musicología tal vez bien diferencial entre, por una parte, el mecanismo de repetición cuya función canónica es la de imitar (lo anterior sin olvidar los preceptos ortodoxos de la antigua mimesis), confiriendo a la melodía dominante su irrefutable especificidad de ideal y, por la otra, el organon, es decir el principio que rige, en esta primera etapa del desarrollo de la polifonía, la concepción de acompañamiento de una melodía principal. Esta función de acompañamiento y no de imitación es determinante para la his-

20 "La imagen de la polifonía y del contrapunto sólo indica los nuevos problemas que surgen cuando la estructura de la novela sale de la unidad monológica habitual, de la misma manera como, en la música, aparecieron nuevos problemas cuando se rebasó el estadio del monovocalismo. Pero los materiales de la música y de la novela son demasiado diferentes como para no hacer más que una comparación aproximativa de metáfora" (Bakhtine 1970, 53). 
toria de la teoría musical, ya que la consonancia es un fenómeno cambiante que encierra, además, el potencial de poder convertirse poco a poco en disonancia según las leyes de la evolución de la escucha. Se trata de un hecho que amerita no ser descuidado cuando se sabe que las técnicas de composición del contrapunto se orientan progresivamente hacia una mayor libertad de movimiento melódico, hacia la posibilidad de diversificación vocal en relación con el tema musical principal, hasta desembocar en la polifonía propiamente dicha. Y, nota interesante, es en el siglo XIV, en España, cuando el organum adquiere el sentido general de polifonía; "canto de órgano" en castellano significa, ni más ni menos, que canto polifónico. En relación con la noción de canon literario, la contrapropuesta que afirma la de un organon sugiere posibilidades de pensar el trabajo intelectual y discursivo en términos de una cultura que "canta" o "habla" la historia con el fin de abrir ésta a diferentes "voces" coexistiendo en sus interacciones. Esto significa reconocer la función del trabajo del discurso en el seno de su propia actualización sociohistórica, y no únicamente en relación con la historia (patriarcal) ya hecha. Se puede entonces concebir un registro de prácticas sobre una base diferencial que, al distanciarse de la práctica dominante lleva a subrayar el carácter fundamentalmente secular del discurso feminista cuando se comprende éste en una óptica sociocrítica.

Un último detalle, sacado de algunos estudios sobre los orígenes de la polifonía musical, apoya la hipótesis de tal proceso de secularización del discurso. De acuerdo con estos estudios, parecería ser que la polifonía misma tiene sus raíces en la música folklórica tradicional. La división axiológicamente jerarquizada entre una música folklórica unisonante y supuestamente "natural" del orden de una cultura oficiosa, por una parte, y la polifonía "cultural", por la otra, producto de la cultura oficial, no sería más que una pura invención teórica para justificar, es decir legitimar, post factum, el hecho de que, en la 
época gótica, haya sido la Iglesia quien cultivó las técnicas polifónicas de manera más intensa que los trovadores en la música profana. En efecto, es en el seno de la música sagrada litúrgica donde la técnica de organum (justamente a partir de fines del siglo IX) adquiere la función de ornar el canto y de embellecer el repertorio gregoriano. Esta función similar a la de los tropos - en lo musical, la polifonía operaba por medio de la superposición la misma cosa que los tropos en literatura por medio de la interposición - viene a confirmar la canonización precoz del binomio naturaleza/cultura, y esto incluso en un terreno tan abstracto como es el de la teoría musical.

Esta breve excursión en el terreno musical - terreno al que vienen unirse el de las matemáticas y de la mecánica— quisiera ser un modesto ejemplo de ensayo transdisciplinario de literatura comparada. No es ciertamente gratuito si se entiende este tipo de excursión como una proposición sugestiva con respecto al valor cognitivo, transformador, de la práctica cultural. Después de todo, y tal como lo señalan los musicólogos, la emergencia de la polifonía medieval es históricamente el producto de una convergencia de dos hechos musicales, dos técnicas de composición conceptualmente muy diferentes, incluso opuestas: la técnica instrumental y la vocal. Pero hay más, ya que no hay que olvidar que Bajtín mismo nos sugiere que la novela no es más que conjugación e integración (textual) de varios géneros o clases de discurso socioculturales diferentes, literarios o no literarios. Es, por lo demás, el fundamento de su pensamiento a propósito de la emergencia de elementos embrionarios novelescos a fines de la Antigüedad clásica, combinatoria que opone a la que constituía la epopeya. ¿Y qué decir de la música de Gustave Mahler?, contemporáneo, o casi, de Dostoievski, y para quien la verdadera polifonía es el producto de la interacción de sonidos musicales y no musicales, a la manera de los diferentes ruidos producidos en una fiesta foránea que le inspiraron estas afirmaciones (ver 
Malcuzynski 1992, 184-185, 208). La polifonía sirvió a Bajtín no sólo como metáfora conceptual para describir, identificar "narratológicamente" la novela dostoievskiana, sino también para considerar, al concluir de su libro sobre Dostoievski, la novela polifónica como un gran paso adelante, no sólo en la evolución de la prosa novelesca, es decir de todos los géneros que gravitan alrededor de la novela, sino incluso en la evolución del pensamiento estético en general. Nos parece que es posible rebasar el marco del género literario de la "novela" y hablar de una polifonía del pensamiento estético. Ésta es capaz de introducirse en los rincones más secretos del ser humano $\mathrm{y}$, antes que nada, en la conciencia humana y en la esfera dialógica de su ser, que no ofrecen ningún asidero artístico si uno se sitúa desde el punto de vista monológico (Bajtín 1970, 345).

Dejando de lado lo que esto suscita como reflexión a propósito de la concepción misma de la noción de "género" (literario o no), el pensamiento de Bajtín sobre la cultura literaria es, en sí mismo, un discurso teórico sociodinámico, "participativo", "interactivo", eminentemente dialógico y transdisciplinario. Se trata de un discurso compuesto, que se abre y se mueve concretamente entre la filosofía del lenguaje y de la estética, las teorías literarias y las que llamamos en la actualidad culturales (por ejemplo, el carnaval, un espectáculo gestual, sincrético y ritual, nos recuerda a Bajtín); un discurso que se nutre de "préstamos" de diversos saberes, tales como las ciencias "puras" (la biología y la física cuántica, en relación con la noción de cronotopo, por ejemplo) o la teoría musical (las técnicas del contrapunto en composición), préstamos que, en los escritos de Bajtín, cumplen funciones mucho más fundamentales que simplemente metafóricas. Todo su pensamiento dialógico es sociointerdiscursivo en el sentido propio del término; una forma de heterogeneidad que no puede abordarse y comprenderse más que en una perspectiva crítica multidisciplinaria. 
La formulación de la práctica feminista que surge a partir de esta reflexión se presenta entonces bajo la forma de un proyecto heterodoxo que deslegitimiza la brecha contradictoria de la jerarquía canónica entre el discurso cultural y la historia. La tarea interactiva sociocrítica consiste en poner de relieve las posibilidades de engendrar tomas de posición socioideológicas que desenmascaren el sistema mitológico, no sólo de lo "no dicho" de la razón genéricamente determinista, sino también su espejo deformador "no decible". Mi hipótesis es que la práctica feminista comprendida en este sentido, entre todas las prácticas de desritualización y de desfetichización, abordada en su valor específico y no transferible de secularización del discurso normativo, constituye en la actualidad tal vez el más grande poder de emancipación diversificadora, en lo que concierne a las relaciones de fuerza entre la problemática canónica y la pragmática de los saberes en el seno de la producción y de la circulación socioculturales.

\section{Bibliografía}

Authiez-Revuz, Jacqueline, "Hétérogénéité montrée et hétérogénéité constitutive", D. R. L. A. V., 26 (1982), 91-151.

Bakhtine, Mikhaill, La poétique de Dostoëvski, trad. Isabelle Kolitcheff, Paris, Senil, 1970.

—, Eshtétique de la création verbale, trad. Alfredo Aucouturier, Paris, Gallimard, 1984.

—, Toward a Philosophy of the Act, trad. Vadim Liapunov, Austin, Texas University Press, 1993.

- et V. N. Volochinov, Le marxisme et la philosophie du language, trad. Marina Yaguello, Paris, Minuit, 1977.

Bloom, Harold, The Western Canon. The Books and School of the Ages, New York, Harcourt, 1994.

Díaz-Diocaretz, Myriam, y Iris M. Zavala, Breve historia feminista de la literatura española (en lengua castellana), Barcelona, Anthropos, 1993. 
JAMESOn, Fredrick, Political Unconscious, Ithaca, Cornell University Press, 1982.

Kristeva, Julia, "Une poétique ruinée", en Bakhtine, La poétique de Dostö̈evski, Paris, Seuil, 1970, pp. 1-27.

Malcuzynski, M.-Pierrette, "The Sociocritical Perspective and Cultural Studies", Critical Studies, 1:1 (1989), 1-22.

_-, "Mikhail Bakhtin and the Sociocritical Practice", Discours social/Social Discourse, 3:1-2 (1990), 83-97.

—, Entre-dialogues avec Bakhtin ou Sociocritique de la (dé)raison polyphonique, Amsterdam, Atlanta, Rodopi, 1992.

-, "Quelques réflexions sur le discours musical chez Bakhtine", Recherches sémiotiques/Semiotic Inquirí, 18:1-2 (1999), 1-22.

PÊCHEux, Michel, "Analyse du discours, langue et idéologie", Langages, 73 (1975a), 3-6.

_, "Mise au point et perspectives à propos de l'analyse automatique du discourse". En colaboración con C. Fuchs, Langage, 37 (1975b), 7-80.

—, Les vérités de la Palice, Paris, Maspero, 1975c.

Robin, Régine, et Marc Angenot, "L'inscription du discours social dans le texte littéraire", Sociocriticism, 1.1 (1985), 53-82.

Robin, Regine, "Pour une socio-poétique de l'imaginaire social", Discours social / Social Discours, 5:1-2 (1993), 7-32. 